\title{
THE UV LUMINOSITY OF OLD NOVAE
}

P.L. Selvelli - CNR, Astronomical Observatory of Trieste, Italy

A. Cassatella - CNR; ESA IUE Observatory, Spain

A. Bianchini - Astronomical Observatory, Padova, Italy

M. Friedjung - Institut d'Astrophysique, Paris. France

R. Gilmozzi - STSCI - Baltimore, USA

\section{INTRODUCTION}

About 16 old novae are accessible to IUE, but only five (RR Pic, $\mathrm{V} 603 \mathrm{Aql}$, HR Del, $\mathrm{DQ}$ Her, and GK Per) have been observed in the ultraviolet and studied in any detail. Considering that, because of selection effects, only few and the brightest objects have been observed, it not possible to draw general conclusions on the UV luminosity Lov and mass accretion rates $\dot{M}_{a}$ c of old novae. We plan to improve the rather poor statistic by observing in the near future all objects falling above the IUE detection limit of $m(V) \simeq 15$ (i.e. $x$ Ser, DK Lac, DN Gem, and HR Lyr). In the following we present preliminary results for 7 old novae observed with the IUE satellite whitin our observing programmes or obtained from the IUE archive.

\section{THE UV LUMINOSITY AND THE MASS ACCRETION RATE}

For each of the 7 old novae considered here we have derived the integrated UV luminosity Lov in the range 1200-3200, values which are listed in Table 1 together with information on the adopted $E(B-V)$, on the distances and the inclination angles. The distances are from Duerbeck (1983) and the inclinations from Warner (1987) except for CP Pup (not included in his ligt) for which we have used the lower limit of about $30^{\circ}$ given by Duerbeck et al. (1987). Becauge of the uncertainties on the reddening corrections and on the distances, one expects uncertainties of up to $50 \%$ on Lov. Fig. 1 is a plot of the UV luminosity Luv as a function of cos $i$. Despite the paucity of the data, the figure strongly suggests a dependence of Lov on cos i in the sense that high inclination eclipsing objects ( $T$ Aur, BT Mon) have a low luminosity, Lor $\simeq 1$ Lo, while objects seen at low inclination or nearly pole-on tend to have larger Luv of up to about 10 Lo. This latter value can be considered as the "intrinsic" UV luminosity. These findings are in agreement with the conclusions reached by Warner (1987) that the "observed" $M_{v}$ of old novae depends on the inclination angle, while the "intrinsic" $M_{v}$ (at $i \simeq 0$ ) does not vary greatly from star to star.

A direct estimate of the mass accretion rate $\dot{M}_{a c}$ can be obtained if the total accretion luminosity Laik is known. If most of the disk luminosity is emitted in the UV, as seems to be the case in old novae, then Luv is not much smaller than Ldis , and it can be used to provide an estimate of Laik. Under the assumption that Laik $\simeq 2$ Lov, as indicated in Wade's models (1984), our data suggest that 
the "intrisic" disk luminosity of the old novae in our sample is $\simeq$ 20 Lo. A representative value of the mass accretion rate in old novae is therefore $\dot{M}_{\mathrm{c} c} \approx 3 \times 10^{17} \mathrm{gr} / \mathrm{s}$ or $4.5 \times 10^{-9} \mathrm{Mo} / \mathrm{yr}$.

TABLE 1

\begin{tabular}{|c|c|c|c|c|}
\hline Object & $d(p c)$ & $E(B-V)$ & $\cos i$ & $\mathrm{~L}_{\mathrm{Uv}} / \mathrm{L}_{\mathbf{0}}$ \\
\hline V 841 Oph & 855 & 0.30 & 1.0 & 9.5 \\
\hline CP Pup & 1500 & 0.27 & $<0.87$ & 12.0 \\
\hline DI Lac & 895 & 0.15 & $<0.87$ & 1.9 \\
\hline Q Cyg & 1485 & 0.25 & 0.64 & 4.6 \\
\hline V 533 Her & 620 & 0.0 & 0.47 & 0.7 \\
\hline T Aur & 600 & 0.35 & 0.37 & 1.7 \\
\hline BT Mon & 1000 & 0.20 & 0.10 & 0.9 \\
\hline
\end{tabular}

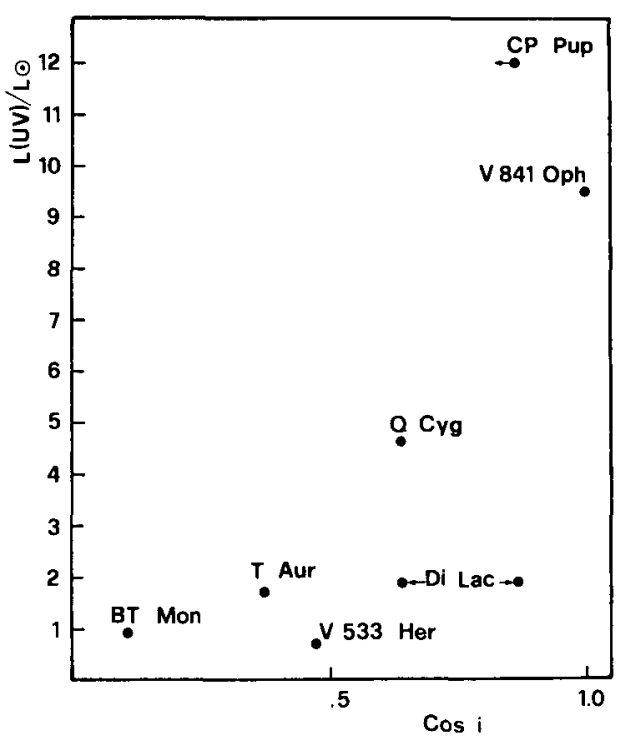

Fig. 1 The ultraviolet luminosity in the range 1200 to 3200 A is plotted as function of the orbital inclination angle for the 7 old novae observed with IUE. 
REMARKS

1) The "intrinsic" UV luminosity suggested by the brightest objects in our sample, Lov $\simeq 10$ Lo, is of the same order of the "observed" UV luminosity of the brightest old novae (Krautter et al. 1981 ).

2) BT Mon is very bright in the optical and, consequently, the value log $\dot{\mathrm{M}}_{\mathrm{ac}} \simeq 7.7$ Mo/yr derived from optical data by Robinson et al. (1982) is surprisingly high. There is no way to reconcile this value with our observations even by using different determinations of reddening and distance.

3) $V 841 \mathrm{Oph}$, one of the oldest nova remnants, is still very bright in the UV, 140 years after the outburst.

4) DI Lac lies outside the mean trend in Fig. 1 for any value of the inclination in the range $30^{\circ}$ to $50^{\circ}$ given by Warner (1987). Note that DI Lac also lies outside the mean Mv vs. cos i trend because of its brighter Mv. Evidently, the disk of DI Lac is rather cool and emits mostly in the optical range.

5) There is no apparent correlation between Luv and parameters such as the orbital period $P$, the rate of decline $t_{3}$, and the time elapsed since the last outburst. X-ray data are available for only 5 objects of our sample (Becker 1989). It is remarkable that the two brightest stars in the UV (CP Pup and V 841 Oph) are also the brightest in the $X$-ray range, while the contrary is true for the three objects ( $T$ Aur, BT Mon, and V 533 Her) which are the weakest in the UV.

\section{REFERENCES}

Becker, R.H.:1989, in "Classical Novae", eds. M.F.Bode, A.Evans, ( J. Wiley), p.215

DuerbeK, H.W.,1983: IAU Coll. No. 80, p.363.

Duerbeck, H.W., Seitter, W.C., and Duemmler, R.: 1987, M.N.R.A.S. 229,653 .

Krautter, J., Klare, G., Wolf, B., Duerbeck, H.W., Rahe, J.,

Vogt, N., and Wargau, W.. 1981, Astron. Astrophys. 102, 337.

Robinson, E.L., Nather, R.E., and Kepler, S.O.: 1982, Astrophys. J. 254,646 .

Wade, R.A.: 1984 , M.N.R.A.S. 208,381 .

Warner, B.: 1986, M.N.R.A.S. 219,751 .

Warner, B.: 1987, M.N.R.A.S. 227,13 . 